Georgian Mathematical Journal

Volume 13 (2006), Number 4, 751-777

\title{
REPRESENTATION OF NUMBERS BY QUADRATIC FORMS. MAIN RESULTS OF THE RESEARCH DONE IN GEORGIA
}

\author{
GEORGE LOMADZE †
}

\begin{abstract}
This is a survey of the main results obtained by the mathematicians of Georgia on the representation of natural numbers by integral quadratic forms before 2000 .
\end{abstract}

2000 Mathematics Subject Classification: 11E20, 11E25.

Key words and phrases: Representation of numbers by quadratic forms, modular form, cusp form, Eisenstein series, singular series.

1. Throughout the 19th century mathematicians were assiduously studying the arithmetic function $r_{s}(n)$ - the number of representations of a natural number $n$ as a sum of $s$ squares of integers. At first they were satisfied with considering only the concrete values for the number of representations. Using the theory of elliptic functions, in 1914 Bulygin [10] for the first time proposed a general method of deriving formulas for $r_{s}(n)$ for all even $s$ when $2 \leq s \leq 24$. These formulas obey a certain general rule. Based on a pure arithmetic approach, later Bessel [7] reproved Bulygin's formulas when $2 \leq s \leq 16$.

Walfisz [146] developed Bessel's arithmetic method further and confirmed Bulygin's formulas for $r_{s}(n)$ when $s=18,20,22,24$.

In his effort to develop general formulas for $r_{s}(n)$ with the aid of modular functions, Mordell ([101], [102]) showed (first for even and then for odd $s$ ) the existence of a function $\chi(\tau)$ such that

$$
\vartheta_{00}^{s}(\tau)=\chi(\tau)+\sum_{k=1}^{(s-1) / 8} A_{k} \vartheta_{00}^{s-8 k}(\tau) \vartheta_{01}^{4 k}(\tau) \vartheta_{10}^{4 k}(\tau),
$$

where $\tau \in \mathbb{C}, \operatorname{Im} \tau>0 ; \vartheta_{i j}(\tau)$ are Jacobi theta-functions, and $A_{k}$ are constants chosen properly. He called the function $\chi(\tau)$ the principal invariant. Mordell went on to give a method for constructing the principal invariant $\chi(\tau)$, separately for even and odd natural numbers $s$. By performing necessary computations, he confirmed once again Bulygin's formulas for $s=10,12,14,16$ and produced new formulas for $s=11,13$ and 15. The so-called additional terms in these formulas are sums taken over all representations of a natural number $n$ as a sum of respectively 3,5 and 7 squares. When $s$ is odd, the so-called main terms in Mordell's formulas contain factors in the form of certain infinite series. Mordell observed that, using Jacobi's well-known identity $\vartheta_{1}^{\prime}=\vartheta_{2} \vartheta_{3} \vartheta_{0}$ [36], it is possible to express the additional term of $r_{9}(n)$ as a sum over all representations of $n$ as sum of 7 squares. 
For the sum of $s$ squares Hardy ([32], [33]) (i) constructed a singular series $\varrho_{s}(n)$, (ii) gave a classical method for its summation, (iii) introduced a function $\theta_{s}(\tau)$ whose Fourier coefficients are the sums of the series $\varrho_{s}(n)$ and, (iv) using modular functions, proved that $\vartheta_{00}^{s}(\tau)=\theta_{s}(\tau)$ for all $3 \leq s \leq 8$. As a consequence of these equalities, one has $r_{s}(n)=\varrho_{s}(n), 3 \leq s \leq 8$. Suetuna ([112], [113]) summed up Hardy's singular series $\varrho_{s}(n)$ for any $s$. Estermann [13], using only the basics of the theory of functions of a complex variable and no modular functions, reproved Hardy's formulas for $r_{s}(n)$.

Lomadze ([53], [54], [55]) developed the method of Estermann and, also using just elements of functions of a complex variable, proved Mordell's identity (1) by a single method both for even and odd numbers $s$. He replaced Mordell's complicated principal invariant $\chi(\tau)$ by Hardy's singular series $\varrho_{s}(n)$. By the results of Suetuna and necessary computations Lomadze ([53], [54], [55]) obtained formulas for $r_{s}(n), 9 \leq s \leq 32$. These formulas coincide with those of Bulygin for even $s, 10 \leq s \leq 24$, and with those of Mordell for odd $s, 11 \leq s \leq 15$. Moreover, in case of odd $s$, Lomadze succeeded for the first time ever in reducing the infinite series contained as factors in the main terms to a finite expression.

The formulas for $r_{s}(n)$ when $s=9,17,25$, obtained by Lomadze on the basis of Mordell's remark, were of minor interest. It was therefore desirable to improve them further. To this end, instead of Jacobi's identity Lomadze later used Smith's equality $\frac{\vartheta_{0}^{\prime \prime}}{\vartheta_{0}}-\frac{\vartheta_{3}^{\prime \prime \prime}}{\vartheta_{3}}=\vartheta_{2}^{4}$ [110] and obtained formulas for $r_{s}(n)$ with the following advantage over the previous formulas: the new worst additional term is a sum taken over all representations of $n$ as a sum of $s-4$ squares as opposed to the previous version of $s-2$ squares [76].

2. Let $r(n ; f)$ denote the number of representations of a natural number $n$ by a positive integral quadratic form $f=f\left(x_{1}, x_{2}, \ldots, x_{s}\right)$. Finding an exact formula for the function $r(n ; f)$ means (i) constructing a singular series $\varrho(n ; f)$ that corresponds to the quadratic form $f$, (ii) finding its sum, and (iii) constructing functions

$$
\left.X_{k}(\tau ; f)=\sum_{n=1}^{\infty} \nu_{k}(n ; f) Q^{n} \quad \text { (here and in what follows } Q=\exp (2 \pi i \tau)\right)
$$

which are regular for $\operatorname{Im} \tau>0$, so that the following equality holds for any natural number $n$ and properly chosen constants $A_{k}$ :

$$
r(n ; f)=\varrho(n ; f)+\sum_{k=1}^{\omega} A_{k} \nu_{k}(n ; f)
$$

with $\omega$ being a finite number depending only on $f$. It is known that

$$
\vartheta(\tau ; f)=1+\sum_{n=1}^{\infty} r(n ; f) Q^{n}
$$

where

$$
\vartheta(\tau ; f)=\sum_{x_{1}, \ldots, x_{s}=-\infty}^{\infty} Q^{f\left(x_{1}, \ldots, x_{s}\right)}
$$


is the theta-series corresponding to the quadratic form $f$. Therefore if the function

$$
\theta(\tau ; f)=1+\sum_{n=1}^{\infty} \varrho(n ; f) Q^{n}
$$

is regular for $\operatorname{Im} \tau>0$, then the arithmetic equality (2) is evidently equivalent to the functional one:

$$
\vartheta(\tau ; f)=\theta(\tau ; f)+\sum_{k=1}^{\omega} A_{k} X_{k}(n ; f) .
$$

The term 'exact formula' is essentially due to Mordell because (1) is a particular case of equality (3). In what follows functions of the form $\theta(\tau ; f)$ will be called the main functions, and those of the form $X_{k}(n ; f)$ - the additional functions. Further, the coefficients of their Fourier series expansions will be called the main and additional terms, respectively.

It is known that if the quadratic form $f$ is of one-class genus, then the additional term is zero and, consequently, for any natural $n$ formula (2) becomes

$$
r(n ; f)=\varrho(n ; f) .
$$

2.1. It is quite natural that, while studying the sums of squares of integers, mathematicians also got interested in diagonal integral positive primitive quadratic forms

$$
f=\left\{a_{1}, a_{2}, \ldots, a_{s}\right\}=a_{1} x_{1}^{2}+a_{2} x_{2}^{2}+\cdots+a_{s} x_{s}^{2} .
$$

For example, Liouville alone published up to 100 short papers in which he presented without proof a lot of formulas for the number of representations of $n$ by quadratic forms of type (4) mainly in four and six variables (see, e.g., [43][52]). All his formulas are expressed in terms of various arithmetic functions of divisors of the natural number being represented. Some of Liouville's formulas have also additional terms with a simple arithmetic meaning. Namely, they are sums taken over certain representations of integers by binary quadratic forms.

Walfisz developed an elementary method for obtaining exact formulas for the number of representations of a natural number by integral positive quaternary quadratic forms of special kind. This method, now known as the Jacobi-Walfisz method, is a further development of an elementary method of Jacobi. It is based on some trigonometric identities (also due to Walfisz) obtained by simple transformations of infinite series. Using his method, Walfisz ([147], [148], [149]) gave exact formulas for the number of representations of a natural number by 73 quaternary quadratic forms, a big part of which were early published by Liouville without proof. Some of Walfisz' formulas were beyond reach of the previous methods (see also [61]).

Using Hardy-Littlewood's method, Kloosterman [41] defined and summed up a singular series corresponding to the quadratic form $f=\{1,1, a, a\}$, where $a$ is any prime or double prime number, and proved that the corresponding function $\theta(\tau ; 1,1, a, a)$ is regular for $\operatorname{Im} \tau>0$. Therefore the function $\theta(\tau ; 1,1, a, a)$ can 
be used as the main function. Then, individually for $a=5,6,7$, he determined the additional functions as products of certain Jacobi theta-functions and, using modular functions, proved identities of type (3). When $a=3$, the additional function is identically zero because the quadratic form $f=\{1,1,3,3\}$ belongs to one-class genus. one can use a method by Kloosterman for other quaternary quadratic forms of type (4). However, for an individual quadratic form that method requires complex formulas from the theory of Jacobi theta-functions and, moreover, involves laborous calculations connecting with studying the behavior of the corresponding modular functions in their fundamental domains. Also, the mentioned method cannot be used for binary and ternary quadratic forms of type (4).

Maass [99] was the first to develop a general method, based on modular functions and Jacobi theta-functions, for obtaining exact formulas for the number of representations of a natural number by ternary quadratic forms of type (4). But Maass' investigation was restricted to the quadratic forms of one-class genera and, moreover, with only square-free coefficients.

Using the Hecke theory of entire modular forms and theta-functions with characteristics, Streefkerk [111] obtained exact formulas for the number of representations of an integer as a sum of certain generalized polygonal numbers.

2.2. Combining and further developing the afore-mentioned methods of Hardy, Mordell, Hecke, Kloosterman, Maass and Streefkerk, Lomadze [56] worked out a uniform general way for obtaining exact formulas for the number of representations of a natural number by any quadratic form of type (4), independently of the number of variables and the genus. To this end, he (similarly to Hardy) defined the following singular series for the quadratic form $f=\left\{a_{1}, a_{2}, \ldots, a_{s}\right\}$ :

$$
\varrho\left(n ; a_{1}, a_{2}, \ldots, a_{s}\right)=\frac{\pi^{s / 2}}{\Gamma(s / 2) \Delta^{1 / 2}} n^{s / 2-1} \sum_{q=1}^{\infty} q^{-s} \sum_{\substack{h \bmod q \\(h, q)=1}} e\left(-\frac{h n}{q}\right) \prod_{k=1}^{s} S\left(a_{k} h, q\right),
$$

where $\Delta$ is the determinant of $f, \Gamma(s / 2)$ is the gamma-function, and $S\left(a_{k} h, q\right)$ is the Gaussian sum. Lomadze also introduced the function

$$
\theta\left(\tau ; a_{1}, a_{2}, \ldots, a_{s}\right)=1+\sum_{n=1}^{\infty} \varrho\left(n ; a_{1}, a_{2}, \ldots, a_{s}\right) Q^{n} .
$$

It is a regular function for $\operatorname{Im} \tau>0$ and $s>4$ because in this region the singular series $\varrho\left(n ; a_{1}, a_{2}, \ldots, a_{s}\right)$ is absolutely convergent. But for $s \leq 4$ Lomadze (similarly to Hecke and Streefkerk) introduced an auxiliary variable $z$ and considered the function

$$
\psi\left(\tau, z ; a_{1}, a_{2}, \ldots, a_{s}\right)=1+\frac{(i / 2)^{s / 2}}{2 \Delta^{1 / 2}} \sum_{\substack{q ; H=-\infty \\(H, q)=1}}^{\infty} i^{s(\operatorname{sgn} q-1) / 2} \frac{\prod_{k=1}^{s} S\left(-a_{k} H \operatorname{sgn} q,|q|\right)}{q^{s / 2}(q \tau+H)^{s / 2}|q \tau+H|^{z}}
$$


where $\sum^{\prime}$ means that the sum has no terms with $q=0$. This function is regular for $\operatorname{Re} z>2-\frac{s}{2}$ and fixed $\tau$.

In order to illustrate the method, from quaternary quadratic forms of kind (4), Lomadze [56] considered arbitrary quadratic forms $f=\left\{a, a, a^{\prime}, a^{\prime}\right\}, f=$ $\left\{a, a, a, a^{\prime}\right\}$ and proved that (i) the functions $\psi\left(\tau, z ; a, a, a^{\prime}, a^{\prime}\right)$ and $\psi\left(\tau, z ; a, a, a, a^{\prime}\right)$, which, by definition, are regular for $\operatorname{Re} z>0$, can be analytically extended in a neighborhood of $z=0$; (ii) $\theta\left(\tau ; a, a, a^{\prime}, a^{\prime}\right)=$ $\left.\psi\left(\tau, z ; a, a, a^{\prime}, a^{\prime}\right)\right|_{z=0}$ and $\theta\left(\tau ; a, a, a, a^{\prime}\right)=\left.\psi\left(\tau, z ; a, a, a, a^{\prime}\right)\right|_{z=0} ;$ (iii) the functions $\theta\left(\tau ; a, a, a^{\prime}, a^{\prime}\right)$ and $\theta\left(\tau ; a, a, a, a^{\prime}\right)$ are regular for $\operatorname{Im} \tau>0$ and also are the modular forms of weight 2 and level $4 a a^{\prime}$. Both functions are used by him as main functions. Further, using Hardy-Kloosterman's method he obtained computationally convenient formulas to compute sums of the series $\theta\left(\tau ; a, a, a^{\prime}, a^{\prime}\right)$ and $\theta\left(\tau ; a, a, a, a^{\prime}\right)$. Finally, for each concrete quadratic form he individually found additional functions in the form of sums of products of theta functions with characteristics which are cusp forms of the same level and weight as the corresponding main functions; using the theory of entire modular forms he proved identities of type (3). This combined method needs long calculations, but they are quite elementary and standard. This method does not need any longer the construction of fundamental regions which in turn need difficult calculations. In particular, Lomadze ([56], [57], [62]) obtained exact formulas for the number of representations of a natural number by 13 concrete quaternary quadratic forms of type (4).

Using the same method, Beridze [2] obtained exact formulas for the number of representations of a natural number by 4, Gelashvili [15] by 3, Gogishvili [17] by 4 concrete quaternary quadratic forms of type (4). Among quadratic forms of type (4), Gongadze [29] considered quadratic forms $f=\left\{1, a_{1}, a_{2}, a\right\}$, where $a$ is the least common multiple of $a_{1}$ and $a_{2}$; for the sum of singular series $\rho\left(n ; 1, a_{1}, a_{1}, a\right)$ corresponding to these quadratic forms she obtained computationally convenient formulas and derived exact formulas for the number of representations of a natural number by 5 concrete quadratic forms of the same type.

In order to illustrate the method in the case of quadratic forms with six variables of type (4), Lomadze ([64], [65]) considered the quadratic form

$$
f=\left\{a_{1}, a_{1}, a_{2}, a_{2}, a_{3}, a_{3}\right\} .
$$

He proved that the function $\theta\left(\tau ; a_{1}, a_{1}, a_{2}, a_{2}, a_{3}, a_{3}\right)$ is the entire modular form of level $4 a$ ( $a$ is the least common multiple of $a_{1}, a_{2}$ and $a_{3}$ ) and weight 3 . For the sums of singular series corresponding to this quadratic form, using again HardyKloosterman's method Lomadze obtained computationally convenient formulas, for each concrete form found additional functions in the form of the sum of products of theta-functions with characteristics, and proved identities of type (3). In particular, he obtained exact formulas for the number of representations of a natural number by 14 concrete quadratic forms of such kind. In the same manner, Beridze and Gogishvili [6] obtained exact formulas for the number of representations of a natural number by the quadratic forms $\{1,1,1,1,1,7\}$ and 
$\{1,7,7,7,7,7\}$. By the same method Beridze [5] considered the quadratic forms with eight variables

$$
f_{1}=\left\{a, a, a, a, a^{\prime}, a^{\prime}, a^{\prime}, a^{\prime}\right\} \quad \text { and } \quad f_{2}=\left\{a, a, a^{\prime}, a^{\prime}, a^{\prime}, a^{\prime}, a^{\prime}, a^{\prime}\right\} .
$$

For the sums of the corresponding singular series she obtained computationally convenient formulas and, for each concrete quadratic form, determined an additional term. She also showed the existence of identities of type (3) and derived exact formulas for the number of representations of a natural number by four concrete forms of type $f_{1}$ and by eight concrete forms of type $f_{2}$. The quadratic forms studied by the Georgian mathematicians belong to multi-class genera.

Now let us consider the quadratic forms $f=\left\{a_{1}, a_{2}\right\}$. The singular series corresponding to these quadratic forms are divergent. Therefore, unlike the case of quadratic forms with $s \geq 4$ variables, one cannot say that $\theta\left(\tau ; a_{1}, a_{2}\right)$ generates a singular series of the quadratic form $f=\left\{a_{1}, a_{2}\right\}$. But Lomadze ([60], [63]) proved that the function $\psi\left(\tau, z ; a_{1}, a_{2}\right)$ which, by definition, is regular for $\operatorname{Re} z>1$, can be analytically extended in a neighborhood of $z=0$. Accordingly, he defined the function $\theta\left(\tau ; a_{1}, a_{2}\right)$ by the equality

$$
\theta\left(\tau ; a_{1}, a_{2}\right)=\left.\psi\left(\tau, z ; a_{1}, a_{1}\right)\right|_{z=0} .
$$

Furthermore, using the lemmas, essentially due to Streefkerk, Lomadze proved that the so defined function $\theta\left(\tau ; a_{1}, a_{2}\right)$ is an entire modular form of level $4 a_{1} a_{2}$ and weight 1 . By the functional equation of the Dirichlet $L$-function and some results of Hecke, he determined the Fourier expansion of $\theta\left(\tau ; a_{1}, a_{2}\right)$ :

$$
\theta\left(\tau ; a_{1}, a_{2}\right)=2+\sum_{n=1}^{\infty} \rho\left(n ; a_{1}, a_{2}\right) Q^{n} .
$$

Here $\rho\left(n ; a_{1}, a_{2}\right)$ simply denotes the Fourier coefficient of $\theta\left(\tau ; a_{1}, a_{2}\right)$ and not a singular series. Lomadze took the function $\frac{1}{2} \theta\left(\tau ; a_{1}, a_{2}\right)$ as the main function and its Fourier coefficient $\frac{1}{2} \rho\left(n ; a_{1}, a_{2}\right)$ as the main term. Next, he obtained computationally convenient formulas for computing the values of $\rho\left(n ; a_{1}, a_{2}\right)$. We will call the function $\rho\left(n ; a_{1}, a_{2}\right)$ a "generalized singular series" and its value a "sum of a generalized singular series". Finally, for each quadratic form $f=$ $\left\{a_{1}, a_{2}\right\}$ Lomadze chose an individual additional function again as a sum of products of theta-functions with characteristics and established identities of type (3). For the first time he ([60], [63]) obtained exact formulas for the number of representations of a natural number by eight concrete binary quadratic forms of type (4) belonging to multi-class genera.

In the same lines, Beridze [3] obtained formulas for the number of representations of a natural number by two concrete pairs of binary quadratic forms of type (4). Using the Dirichlet formulas for the number of representations of a natural number by all primitive binary quadratic forms with a negative discriminant, in combination with the formulas of Lomadze for the number of representations of a natural number by diagonal binary quadratic forms, Vepkhvadze [133] obtained exact formulas for the number of representations of a natural number 
by all primitive non-diagonal quadratic forms with discriminants $-44,-68$ and -80 .

Let us now consider the quadratic forms $f=\left\{a_{1}, a_{2}, a_{3}\right\}$. In this case Lomadze [59] proved that the function $\psi\left(\tau, z ; a_{1}, a_{2}, a_{3}\right)$, regular for $\operatorname{Re} z>\frac{1}{2}$ by definition, allows an analytical extension in a neighborhood of the point $z=0$. Assuming (similarly to the case $\left.f=\left\{a_{1}, a_{2}\right\}\right)$ that $\theta\left(\tau ; a_{1}, a_{2}, a_{3}\right)=\left.\psi\left(\tau, z ; a_{1}, a_{2}, a_{3}\right)\right|_{z=0}$, he proved that the function $\theta\left(\tau ; a_{1}, a_{2}, a_{3}\right)$ is regular for $\operatorname{Im} z>0$ and determined the Fourier expansion

$$
\theta\left(\tau ; a_{1}, a_{2}, a_{3}\right)=1+\sum_{n=1}^{\infty} \rho\left(n ; a_{1}, a_{2}, a_{3}\right) Q^{n} .
$$

The weight of the function $\theta\left(\tau ; a_{1}, a_{2}, a_{3}\right)$ is $\frac{3}{2}$. In order to avoid the study of entire modular forms with half-integral weight, Lomadze considered the function

$$
\begin{aligned}
\psi(\tau)= & \prod_{k=1}^{3} \vartheta_{00}\left(\tau ; 0,2 a_{k}\right)-\theta\left(\tau ; a_{1}, a_{2}, a_{3}\right) \\
& -\sum_{k=1}^{\omega} A_{k} \prod_{i=1}^{3} \vartheta_{g_{k_{i}} h_{k_{i}}}\left(\tau ; 0,2 N_{k_{i}}\right),
\end{aligned}
$$

where $A_{k}$ is any constant and $\vartheta_{00}\left(\tau ; 0,2 a_{k}\right), \vartheta_{g_{k_{i}} h_{k_{i}}}\left(\tau ; 0,2 N_{k}\right)$ are theta-functions with characteristics. He proved that if the function $\psi(\tau)$ satisfies certain conditions, then $\psi^{4}(\tau)$ is an entire modular form of level $4 a$ ( $a$ is the least common multiple of $\left.a_{1}, a_{2}, a_{3}\right)$ and weight 6 . Lomadze also proved that the singular series corresponding to the quadratic form $f=\left\{a_{1}, a_{2}, a_{3}\right\}$ is convergent but not absolutely convergent. Therefore the Fourier coefficients $\rho\left(n ; a_{1}, a_{2}, a_{3}\right)$ of the function $\theta\left(\tau ; a_{1}, a_{2}, a_{3}\right)$ are in fact singular series of the quadratic form $f=\left\{a_{1}, a_{2}, a_{3}\right\}$ and $\theta\left(\tau ; a_{1}, a_{2}, a_{3}\right)$ is the generating function just like in the case where the number of variables of the quadratic form $f=\left\{a_{1}, a_{2}, \ldots, a_{s}\right\}$ is more than 3. He obtained convenient formulas for computing the sum of the series $\rho\left(n ; a_{1}, a_{2}, a_{3}\right)$ ([70], [58], [59], [68]). Consequently, for the quadratic forms $f=\left\{a_{1}, a_{2}, a_{3}\right\}$ the function $\theta\left(\tau ; a_{1}, a_{2}, a_{3}\right)$ is the main function. Finally, for each quadratic form $f=\left\{a_{1}, a_{2}, a_{3}\right\}$ Lomadze chose additional functions in the form of sums of products of such theta-functions with characteristics that the function defined by (7) is identically equal to zero. For the first time he ([67], [78]) obtained formulas for the number of representations of a natural number by 8 concrete ternary quadratic forms of type (4) and of multi-class genera.

Using the same method, Sulakvelidze [114] obtained exact formulas for the number of representations of a natural number by 6 concrete ternary quadratic forms of type (4) belonging to multi-class genera.

Remark. Throughout the section we followed the convention that (i) if the number of variables of a quadratic form $f$ is $s \geq 3$, then $\theta(\tau ; f)$ denotes the generating function of the singular series $\rho(n ; f)$ and (ii) if $s=2$, then $\theta(\tau ; f)=$ $\left.\psi(\tau, z ; f)\right|_{z=0}$ and $\rho(n ; f)$ is its Fourier coefficient called the "generalized singular series". 
2.3. Lomadze's general combined method for obtaining exact formulas for the number of representations of a natural number by diagonal binary quadratic forms was extended by Vepkhvadze ([131], [132], [135], [136]) to all (not necessarily diagonal) integral positive binary quadratic forms. To this end, he first established several relations between double Gaussian sums, defined a function $\psi(\tau, z ; f)$ (similar to the function defined in Subsection 2.2) for non-diagonal binary quadratic forms $f$ - separately for the cases of even and odd discriminants, and then proved that the latter function can be extended analytically in a neighborhood of $z=0$. Next Vepkhvadze obtained convenient formulas for computing the sum of the corresponding generalized singular series and derived exact formulas for the number of representations of a natural number by 23 particular non-diagonal binary quadratic forms of multi-class genera. His results also include exact formulas for the number of representations of a natural number by 3 quadratic forms of the same genus. Such formulas were earlier obtained by Van der Blij [8], but his method does not extend to exact formulas for the number of representations by other binary quadratic forms.

Jones and Pall [37] proved that there exist only 82 primitive positive quadratic forms of type $f=\left\{a_{1}, a_{2}, a_{3}\right\}$ and of one class genus. Using the formulas for the singular series $\rho\left(n ; a_{1}, a_{2}, a_{3}\right)$, Lomadze ([59], [68], [70]) derived formulas for the number of representations of a natural number by all these 82 quadratic forms. Some of these formulas, using a different method, were previously obtained by Uspenskij ([125], [126]) and, also, by Maass [99]. But Lomadze's formulas are all stated in a computationally very convenient form. Further, these formulas directly imply the results of Dickson and Jones (see, e.g., [11, pp. 111-113]) on the arithmetic progressions whose terms are exactly the numbers not representable by the mentioned quadratic forms.

Lomadze's general combined method for deriving exact formulas for the number of representations of a natural number by diagonal ternary quadratic forms was extended by Sulakvelidze ([117], [116], [119]) to any ternary non-diagonal integral positive quadratic forms. To this end, using an argument of Vepkhvadze ([135], [136]), he (i) established several important relations between triple Gaussian sums, (ii) proved that the singular series, corresponding to any non-diagonal ternary positive quadratic form, is convergent but not absolutely convergent, (iii) obtained computationally convenient formulas for the sum of this series, and (iv) derived exact formulas for the number of representations of a natural number by 21 particular non-diagonal ternary quadratic forms ( 5 of which are of one-class and the rest 16 of two-class genera). He also determined those arithmetic progressions whose terms and only those are not representable by the 5 afore-mentioned quadratic forms. As for the other 16 quadratic forms (of two-class genera), he only managed to determine those arithmetic progressions whose terms are not representable by these forms.

3.1. The exact formulas for the number of representations of a natural number by quadratic forms of multi-class genera with two, three, four, six and eight variables, obtained by Kloosterman and the Georgian mathematicians, are not 
of the Liouville type. The arithmetic nature of the sums of singular series, corresponding to positive integral quadratic forms, is straightforward - these sums are simple arithmetic functions depending only on the divisors of the natural numbers being represented. But the additional terms of these exact formulas are defined only as Fourier coefficients of the products of Jacobi theta-functions or theta-functions with characteristics. The clarification of the arithmetic nature of these additional terms is a big challenge. Consequently, the problem was posed to define additional functions whose Fourier coefficients have a simple arithmetic meaning. Lomadze [64] proved that for a natural number $N$ the function $\prod_{k=1}^{s} \vartheta_{g_{k} h_{k}}\left(\tau ; 0,2 N_{k}\right)$ is an entire modular form of level $4 N$ and weight $-\frac{s}{2}$ provided that $2|s, 2| g_{k}, N_{k} \mid N(k=1,2, \ldots, s)$ and $4 \mid N \sum_{k=1}^{s} \frac{h_{k}^{2}}{N_{k}}$. This result made it possible to reveal the arithmetic meaning of the additional terms for the exact formulas that were constructed by him for the above-mentioned 14 quadratic forms in six variables. These additional terms turned out to be sums over certain representations of integers by quaternary quadratic forms. Vepkhvadze [134] investigated quadratic forms of type $f=\left\{a, a, a, a^{\prime}, a^{\prime}, a^{\prime}\right\}$, summed up the corresponding singular series, and obtained computationally convenient formulas for its sum. Namely, for 3 quadratic forms of this type he obtained exact formulas similar to those by Lomadze [64]. Using the same result of Lomadze, Beridze [4] revealed the arithmetic meaning of the additional terms of the exact formulas for the afore-mentioned 12 concrete quadratic forms with eight variables of type (6). The result was similar to that for six variables. She derived also 4 exact formulas for quadratic forms $f=\left\{a_{1}, a_{2}, \ldots, a_{8}\right\}$ where the coefficients of an odd number of variables are equal to 1 and the rest coefficients are equal to 2 .

For quaternary quadratic forms the question remained open. Also, as we saw in the case of quadratic forms with six and eight variables, the obtained formulas are not of the Liouville type. The only exceptions are Vepkhvadze's ([130], [129]) Liouville type formulas for the number of representations of a natural number by the quadratic forms $\{1,1,1,1,4,4\},\{1,1,4,4,4,4\}$ and $\{1,1,2,2,4,4\}$, first published without proof by Liouville ([49], [50], [51]), and also the quadratic form $\{1,1,1,3,3,3\}$. In the latter case an incomplete formula was earlier published by Uspenskij [125]. Vephkhvadze's all four formulas are of the Liouville type and based on the above-mentioned result of Lomadze.

3.2. In order to fill the existing gap, Lomadze [71] studied how the first and second derivatives of theta-functions with characteristics behave with respect to the action of a linear substitution of the congruence group $\Gamma_{0}(4 N)$. This offered a possibility to construct cusp forms whose Fourier coefficients have a simple arithmetic meaning. Namely, Lomadze proved that if certain conditions are met (similar to those mentioned in Subsection 3.1), then for a natural number $N$ :

(i) the function $\vartheta_{g_{1} h_{1}}^{\prime}\left(\tau ; 0,2 N_{1}\right) \vartheta_{g_{2} h_{2}}\left(\tau ; 0,2 N_{2}\right)$ is a cusp form of weight 2 and character $\chi(\delta)=\left(\frac{\Delta}{|\delta|}\right)$ with respect to the congruence group $\Gamma_{0}(4 N)$ (here $\Delta$ 
refers to the determinant of any integral positive quaternary quadratic form for which this function is used, and $(\div)$ here and in what follows stands for Jacobi symbol if not being defined otherwise),

(ii) the functions

$$
\vartheta_{g_{1} h_{1}}^{\prime}\left(\tau ; 0,2 N_{1}\right) \vartheta_{g_{2} h_{2}}^{\prime}\left(\tau ; 0,2 N_{2}\right)
$$

and

$$
\frac{1}{N_{1}} \vartheta_{g_{1} h_{1}}^{\prime \prime}\left(\tau ; 0,2 N_{1}\right) \vartheta_{g_{2} h_{2}}\left(\tau ; 0,2 N_{2}\right)-\frac{1}{N_{2}} \vartheta_{g_{1} h_{1}}\left(\tau ; 0,2 N_{1}\right) \vartheta_{g_{2} h_{2}}^{\prime \prime}\left(\tau ; 0,2 N_{2}\right)
$$

are cusp forms of weight 3 and character $\chi(\delta)=\operatorname{sgn} \delta\left(\frac{-\Delta}{|\delta|}\right)$ with respect to congruence group $\Gamma_{0}(4 N)$ ( $\Delta$ is the determinant of any positive integral quadratic form with six variables for which this function is used).

Using the function (i), Lomadze ([66], [69]) obtained exact Liouville type formulas for the number of representations of a natural number by the quadratic forms $\{1,1,1,6\},\{1,1,1,7\},\{1,1,1,9\},\{1,1,1,15\},\{1,1,1,24\},\{1,1,1,32\}$ and $\{3,8,8,8\}$; Beridze [2] did the same for the quadratic forms $\{1,6,6,6\}$, $\{1,7,7,7\},\{2,2,2,3\}$ and $\{2,3,3,3\}$; and Gongadze [30] for the quadratic forms $\{1,2,32,32\},\{1,8,32,32\}$ and $\{1,32,32,32\}$.

The described method for producing Liouville type formulas was further extended by Gogishvili ([19], [21]) to any diagonal integral quaternary positive quadratic forms and obtained handy formulas for the sums of the singular series' corresponding to these forms. He also showed in this general case that the function $\theta\left(\tau ; a_{1}, a_{2}, a_{3}, a_{4}\right)$, which is an entire modular form of level $4 a$ ( $a=$ $\left.\operatorname{lcm}\left(a_{1}, a_{2}, a_{3}, a_{4}\right)\right)$ and weight 2 , can serve as a main function. For several particular quadratic forms individually, again using the function (i), Gogishvili ([20], [21]) found additional functions and proved equalities of type (3). In particular, he was the first to obtain Liuoville type exact formulas for the number of representations of a natural number by the quadratic forms $\{1,1,2,12\}$, $\{1,1,16,32\},\{1,2,8,32\},\{1,3,36,36\},\{1,4,9,36\},\{1,4,16,32\},\{1,8,8,32\}$, $\{1,12,36,36\},\{1,16,16,32\},\{3,4,9,36\}$ and $\{4,9,12,36\}$. Using these results by Gogishvili, the function (1) and his own results on non-diagonal binary quadratic forms, Vepkhvadze [137] obtained Liuoville type exact formulas for the number of representations of a natural number by 3 non-diagonal quaternary quadratic forms which are the sums of two binary non-diagonal quadratic forms.

Using the functions (ii), Lomadze [73] obtained Liouville type exact formulas for the number of representations of a natural number by quadratic forms $\{1,1,1,5,5,5\},\{1,1,1,7,7,7\}$ and $\{1,1,1,8,8,8\}$. He computed the sums of the corresponding singular series with the use of Vepkhvadze's [134] formulas mentioned in Subsection 3.1.

Malyshev [100] constructed, studied and represented in terms of an infinite product the most general Hardy-Littlewood singular series $H_{g ; b_{1}, \ldots, b_{s}}(f ; n)$ that corresponds to the integral positive quadratic form $f$ with $s \geq 4$ variables. Malyshev also showed that by means of this series the main term of an asymptotic formula for the number of all entire representations $\left(x_{1}, x_{2}, \ldots, x_{s}\right)$ of $n$ by $f$ can be expressed if $x_{i} \equiv b_{i}(\bmod g)(i=1, \ldots, s)$. Using some arguments 
of Streefkerk, Beridze [1] summed up this series, namely: if it is assumed that the determinant of the quadratic form $f$ is $\Delta=r^{2} \omega$ ( $\omega$ is square-free) and $v=\prod_{\substack{p \mid n \\ p \nmid 2 \Delta g}} p^{w}$, then for even $s$

$$
\begin{aligned}
H_{g ; b_{1}, \ldots, b_{s}}(f ; n)= & \chi(2) \prod_{\substack{p \mid \Delta g \\
p>2}} \chi(p) \prod_{\substack{p \mid r g \\
p>2}}\left(1-\left(\frac{(-1)^{s / 2} \omega}{p}\right) p^{-s / 2}\right)^{-1} \\
& \times L\left(\frac{s}{2},(-1)^{s / 2} \omega\right) \sum_{d \mid v}\left(\frac{(-1)^{s / 2} \Delta}{d}\right) d^{1-s / 2}
\end{aligned}
$$

where $\left(\frac{(-1)^{s / 2} \omega}{p}\right)$ is the Legendre symbol. She also obtained a similar formula for odd $s$. Using the functions (ii), Beridze and Gogishvili [6] obtained Liouville type exact formulas for the number of representations of a natural number by the quadratic forms $\{1,1,1,1,1,5\},\{1,5,5,5,5,5\}$ and $\{1,8,8,8,8,8\}$. The sums of singular series corresponding to these quadratic forms were calculated by means of formula (8). Sulakvelidze [121] used the functions (ii) to obtain Liouville type formulas for the number of representations of a natural number by eight non-diagonal quadratic forms in six variables. He calculated the sums of the singular series corresponding to these quadratic forms by means of (8).

3.3. Jones and Pall [37] proved that there exist only 20 so-called regular primitive integral positive quadratic forms

$$
f=\left\{a_{1}, a_{2}, a_{3}\right\}=a_{1} x_{1}^{2}+a_{2} x_{2}^{2}+a_{3} x_{3}^{2},
$$

which belong to multi-class genera. They also discovered such arithmetic progressions related to the genera of these quadratic forms, whose terms are not representable by the corresponding form $f$. They also gave all other quadratic forms from the afore-mentioned multi-class genera, which are the so-called semiregular primitive quadratic forms. Below we shall use the following notation:

$$
\begin{gathered}
g=\left\{c_{11}, c_{22}, c_{33}, c_{23}, c_{13}, c_{12}\right\} \\
=c_{11} x_{1}^{2}+c_{22} x_{2}^{2}+c_{33} x_{3}^{2}+2 c_{23} x_{2} x_{3}+2 c_{13} x_{1} x_{3}+2 c_{12} x_{1} x_{2} .
\end{gathered}
$$

Jones and Pall also found the integers which, like the numbers of the aforementioned arithmetic progressions, are not representable by the corresponding form $g$. Lomadze ([67], [78]) proved that if the function

$$
\psi(\tau)=\prod_{k=1}^{3} \vartheta_{00}\left(\tau ; 0,2 a_{k}\right)-\theta\left(\tau ; a_{1}, a_{2}, a_{3}\right)-A \vartheta_{g h}^{\prime}(\tau ; 0,2 N)
$$

satisfies certain conditions, then for a given natural number $N$ the function $\psi^{4}(\tau)$ is a cusp form of level $4 N$ and weight 6 . He used this result to obtain exact formulas for the number of representations of a natural number by the following six regular quadratic forms $f$ belonging to two-class genera and by 
the corresponding semi-regular quadratic forms $g$ :

$$
\begin{array}{ll}
f_{1}=\{1,1,16\} ; & g_{1}=\{2,2,5,-1,-1,0\} ; \\
f_{2}=\{1,4,16\} ; & g_{2}=\{4,4,5,0,-2,0\} ; \\
f_{3}=\{1,16,16\} ; & g_{3}=\{4,9,9,1,2,2\} ; \\
f_{4}=\{1,8,64\} ; & g_{4}=\{4,8,17,0,-2,0\} ; \\
f_{5}=\{1,3,36\} ; & g_{5}=\{3,4,9\} ; \\
f_{6}=\{1,12,36\}, & g_{6}=\{4,9,12\} .
\end{array}
$$

Formulas for the number of representations of an integer by some of these quadratic forms had been known previously, but all of them were ineffective when used for computations, while the formulas obtained by Lomadze turned out very convenient. Thus, for instance, if $n=2^{\alpha} u(\alpha \geq 0,2 \nmid u), u=s^{2} k$ ( $k$ is square-free), then for $n=s^{2}$

$$
r\left(n, f_{1}\right)=\rho\left(n ; f_{1}\right)+\left(\frac{-1}{s}\right) 2 s, \quad r\left(n, f_{4}\right)=\rho\left(n ; f_{4}\right)+\left(\frac{-2}{s}\right) s,
$$

and for all other values of $n$

$$
r\left(n, f_{1}\right)=\rho\left(n ; f_{1}\right), \quad r\left(n, f_{4}\right)=\rho\left(n ; f_{4}\right) .
$$

The sums of the singular series $\rho\left(n ; f_{i}\right)$ are calculated by Lomadze's formulas which had already been discussed above. Since the quadratic forms $f_{i}$ and the corresponding semi-regular quadratic forms $g_{i}$ belong to the same two-class genera, by a theorem of Siegel $\rho\left(n ; f_{i}\right)=\rho\left(n ; g_{i}\right)$ and the corresponding additional terms differ only in sign. The obtained formulas immediately imply the results of Jones and Pall on the numbers not representable by quadratic forms of type $f$ and $g$.

Sulakvelidze ([117], [119], [120]) obtained exact formulas for the number of representations of a natural number by twelve non-diagonal positive regular and semi-regular ternary quadratic forms, which belong to two-, three- and even four-class genera. The additional terms in his formulas have the same simple arithmetic meaning as in Lomadze's formulas. Using his own formulas, Sulakvelidze established the arithmetic progressions whose terms and only they are not representable by the corresponding quadratic forms.

Watson [150] proved that there exists the so-called universal class of positive integral ternary quadratic forms. It is also known that there is a finite number of universal classes of positive integral quaternary quadratic forms. Sulakvelidze [122] called the genus of integral positive quadratic forms universal if any natural number is representable at least by one quadratic form from this genus. He proved that there exists no universal genus of ternary quadratic forms and there exists an infinite number of universal genera of quaternary quadratic forms. Moreover, he gave an effective method of defining the numbers which are not representable by these genera. 
3.4. It is a well known fact that for any odd natural $n$

$$
\begin{aligned}
& \sum_{\left(6 x_{1}+1\right)^{2}+\left(6 x_{2}+1\right)^{2}+\left(6 x_{3}+1\right)^{2}=3 n}(-1)^{x_{1}+x_{2}+x_{3}}= \begin{cases}\left(\frac{-1}{s}\right) s & \text { if } n=s^{2}, \quad s>0, \\
0 & \text { otherwise, }\end{cases} \\
& \sum_{\substack{x_{1}^{2}+4 x_{2}^{2}+4 x_{3}^{2}=n \\
2 \nmid x_{1}, x_{1}>0}}(-1)^{x_{3}}= \begin{cases}\left(\frac{-1}{s}\right) s & \text { if } n=s^{2}, \quad s>0, \\
0 & \text { otherwise. }\end{cases}
\end{aligned}
$$

Both equalities were proved by means of the theory of elliptic functions, the first of them by Jacobi and the second by Liouville. Venkov ([127], [128]) used his own arithmetic theory of quaternions to show that both equalities are true and that for any odd natural $n$

$$
\sum_{\substack{x_{1}^{2}+x_{2}^{2}+x_{3}^{2}=11 n \\ 2 \nmid x_{1}, 2 \nmid x_{2}, 2 \nmid x_{3} \\ x_{1}>0, x_{2}>0, x_{3}>0}}\left(\frac{11}{x_{1} x_{2} x_{3}}\right)= \begin{cases}-3\left(\frac{-1}{s}\right) s & \text { if } n=s^{2}, \quad s>0, \\ 0 & \text { otherwise. }\end{cases}
$$

Lomadze [84] proved the above three equalities as well as some others by means of the theory of entire modular forms. One of these equalities was

$$
\sum_{\substack{x_{1}^{2}+x_{2}^{2}+x_{3}^{2}=9 n \\ 2 \nmid x_{1}, 2 \nmid x_{2}, 2 \nmid x_{3} \\ x_{1}>0, x_{2}>0, x_{3}>0}}\left(\frac{3}{x_{1} x_{2} x_{3}}\right)= \begin{cases}-3\left(\frac{-1}{s}\right) s & \text { if } n=s^{2}, \quad s>0, \\ 0 & \text { otherwise. }\end{cases}
$$

3.5. Lomadze [90] proved that if for a given natural $N$ the functions

$$
\begin{aligned}
\text { (i) } \psi_{5}(\tau)= & \prod_{k=1}^{5} \vartheta_{00}\left(\tau ; 0,2 a_{k}\right)-\theta\left(\tau ; a_{1}, \ldots, a_{5}\right) \\
& -A \vartheta_{g_{1} h_{1}}^{\prime}\left(\tau ; 0,2 N_{1}\right) \prod_{k=2}^{3} \vartheta_{g_{k} h_{k}}\left(\tau ; 0,2 N_{k}\right), \\
\text { (ii) } \psi_{7}(\tau)= & \prod_{k=1}^{7} \vartheta_{00}\left(\tau ; 0,2 a_{k}\right)-\theta\left(\tau ; a_{1}, \ldots, a_{7}\right) \\
& -A \prod_{k=1}^{2} \vartheta_{g_{k} h_{k}}^{\prime}\left(\tau ; 0,2 N_{k}\right) \vartheta_{g_{3} h_{3}}\left(\tau ; 0,2 N_{3}\right), \\
\text { (iii) } \psi_{9}(\tau)= & \prod_{k=1}^{9} \vartheta_{00}\left(\tau ; 0,2 a_{k}\right)-\theta\left(\tau ; a_{1}, \ldots, a_{9}\right) \\
& -A \prod_{k=1}^{3} \vartheta_{g_{k} h_{k}}^{\prime}\left(\tau ; 0,2 N_{k}\right)
\end{aligned}
$$

with any constant $A$ satisfying certain conditions, then they are cusp forms with the corresponding multiplier systems with respect to the congruence group 
$\Gamma_{0}(4 N)$ and with weights $\frac{5}{2}, \frac{7}{2}, \frac{9}{2}$, respectively. In formula (9) Lomadze assumed that $a_{1}=\cdots=a_{9}$ and chose functions $\vartheta_{g_{k} h_{k}}\left(\tau ; 0,2 N_{k}\right)$ such that the function $\psi_{9}(\tau)$ satisfy the requirements. Thus it became possible to choose a constant $A$ such that the function $\psi_{9}(\tau)$ identically vanishes. For the Fourier coefficients of the expansion of $\psi_{9}(\tau)$ he obtained the formula [87]

$$
r_{9}(n)=\rho_{9}(n)+\frac{32}{17} \sum_{\substack{x_{1}^{2}+x_{2}^{2}+x_{3}^{2}=3 n \\ x_{1}>0, x_{2}>0, x_{3}>0}}\left(\frac{x_{1} x_{2} x_{3}}{3}\right) x_{1} x_{2} x_{3}
$$

where $\left(\frac{x_{1} x_{2} x_{3}}{3}\right)$ is the generalized Legendre symbol and $\rho_{9}(\tau)$ stands for the sum of the singular series that corresponds to the sum of nine squares of integers (cf. [53], [76]). This formula essentially improves Mordell's conjecture that the number of integral representations of a natural number $n$ by the quadratic form $f=x_{1}^{2}+x_{2}^{2}+\cdots+x_{9}^{2}$ can be defined by solving an equation consisting of seven squares.

Not long ago Lomadze [97] obtained exact formulas for the number of representations of a natural number by the quadratic form $x_{1}^{2}+x_{2}^{2}+\cdots+4 x_{9}^{2}$. In this case, too, the problem reduces to solving an integral equation consisting of three squares.

4.1. Throughout this subsection $f$ will denote an integral primitive positive quadratic form with $s$ variables. The question is posed as to the existence of formulas of the type

$$
r(n ; f)=\rho(n ; f)
$$

Rankin [105] proved that such formulas do not exist in particular cases where $f$ is the sum of $s$ squares of integers, $s>8$. Gogishvili [22] extended this result of Rankin to any quadratic form $f$. Namely, he showed that for a quadratic form $f$ with $s \geq 4$ variables there is a constant $C=C(f)$ such that formulas of type (10) do not exist if the determinant $\Delta$ of $f$ is greater than $C$. Hence it follows that there exists only a finite number of those classes of quadratic forms $f$ for which such formulas exist. In particular, all quadratic forms with $s \geq 4$ variables for which $\Delta>C$ belong to multi-class genera.

The following result is also due to Gogishvili [24]: $\rho(n ; f) \rightarrow 0$ as $s \rightarrow \infty$ if the number $n$ representable by $f$ is chosen properly. Such numbers $n$ are constructed explicitly and a formula of type (10) does not exist for any of the quadratic forms $f$ with $s \geq 34$. Later he [25] proved the nonexistence of formulas of type (10) for any quadratic form $f$ with $s$ variables, where $11<s<34$, perhaps except for some quadratic forms with $s=12$ and $s=16$ variables. In addition, Gogishvili [23] showed that for diagonal ternary quadratic forms $f$, formulas of type (10) exist only if these quadratic forms belong to one-class genera.

For $s \geq 4$, the results by Malyshev [100] imply that

$$
\rho(n ; f)=O\left(n^{s / 2-1+\varepsilon} \Delta^{1 / 2}\right)
$$

where $\varepsilon$ is any positive number. 
Gogishvili [27] essentially improved this estimate by proving

$$
\begin{gathered}
\rho(n ; f)=O\left(n \Delta^{-1 / 3} \ln \ln \Delta \ln \ln n\right) \quad \text { if } \quad s=4, \\
\rho(n ; f)=O\left(n^{s / 2-1} \Delta^{-(s-2) / 2(s-1)}\right) \quad \text { if } \quad s \geq 5 .
\end{gathered}
$$

Estimate (11) cannot be further improved since for any $s \geq 5$ there is a quadratic form with $s$ variables for which estimate (11) is unimprovable.

4.2. Let $F(\tau ; f)$ denote a theta-series of a genus containing a primitive integral quadratic form $f$. Siegel [108] proved that if the number of variables of a quadratic form $f$ (both positive-definite and indefinite) is $s>4$, then

$$
F(\tau ; f)=E(\tau ; f)
$$

where $E(\tau ; f)$ is the Eisenstein series defined by

$$
E(\tau ; f)=1+\frac{e^{(2 m-s) \pi i / 4}}{|d|^{1 / 2}} \sum_{q=1}^{\infty} \sum_{\substack{H=-\infty \\(H, q)=1}}^{\infty} \frac{S(f H, q)}{q^{s / 2}(q \tau-H)^{m / 2}(q \bar{\tau}-H)^{(1-m) / 2}} .
$$

Here $\operatorname{Im} \tau>0, m$ and $d$ are respectively the inertia index and the discriminant of $f, S(f H, q)$ is the Gaussian sum. From formula (12) follows the well-known Siegel's theorem [108]: the sum of the singular series corresponding to the quadratic form $f$ is equal to the average number of representations of a natural number by a genus that contains the form $f$.

Later Ramanathan [104] proved that for any primitive integral quadratic form $f$ with $s \geq 3$ variables (except for zero forms with variables $s=3$ and zero forms with variables $s=4$ whose discriminant is a perfect square), there is a function

$$
E(\tau, z ; f)=1+\frac{e^{(2 n-s) \pi i / 4}}{|d|^{1 / 2}} \sum_{q=1}^{\infty} \sum_{\substack{H=-\infty \\(H, q)=1}}^{\infty} \frac{S(f H, q)}{q^{s / 2}(q \tau-H)^{m / 2}(q \bar{\tau}-H)^{(s-m) / 2}|q \tau-H|^{z}},
$$

which he called the Eisenstein-Siegel series and which is regular for any fixed $\tau$ when $\operatorname{Im} \tau>0$ and $\operatorname{Re} z>2-\frac{s}{2}$, analytically extendable in a neighborhood of $z=0$, and that

$$
F(\tau ; f)=\left.E(\tau, z ; f)\right|_{z=0} .
$$

For $s>4$ the function $\left.E(\tau, z ; f)\right|_{z=0}$ coincides with the function $E(\tau ; f)$, and the formula (13) with Siegel's formula (12).

Vepkhvadze ([139], [140]) proved that the function $E(\tau, z ; f)$ is analytically extendable in a neighborhood of $z=0$ also in the case where $f$ is any nonzero integral binary quadratic form (both positive-definite and indefinite) and that

$$
F(\tau ; f)=\left.\frac{1}{2} E(\tau, z ; f)\right|_{z=0} .
$$

As has already been said in Subsection 2, the Georgian mathematicians proved this fact at first only for diagonal positive quadratic forms with $s \geq 2$ variables and for an arbitrary positive binary quadratic form. Ramanathan believed that this result fails to hold for positive and nonzero indefinite binary quadratic forms. 
It follows from (14) that half of "the sum of a generalized singular series" that corresponds to a binary quadratic form is equal to the average number of representations of a natural number by the genus containing this quadratic form. In particular, if a quadratic form belongs to a one-class genus, then for natural $n$

$$
r(n ; f)=\frac{1}{2} \rho(n ; f)
$$

Vepkhvadze [138] also showed the existence of positive integral binary forms which belong to multi-class genera, but for which equality (15) is true.

Gogishvili [26] proved that formulas of type (15) for any natural $n$ exist only for a finite number of classes of integral primitive binary quadratic forms $f$ with integral coefficients. According to him, a formula of type (15) does not exist for any binary form of the genus with the number of classes $\geq 3$.

Van der Blij [9] considered the function

$$
E(\tau ; f, B, g)=\delta+\frac{e^{(2 m-s) \pi i / 4}}{g^{s / 2}|d|^{1 / 2}} \sum_{q=1}^{\infty} \sum_{\substack{H=-\infty \\(H, q)=1}}^{\infty} \frac{S(f H, q, B, g)}{q^{s / 2}(q \tau-H)^{m / 2}(q \bar{\tau}-H)^{(s-m) / 2}},
$$

where $g$ is a natural number, $B=\left(b_{1}, b_{2}, \ldots, b_{s}\right)$ is a one-column matrix with integer entries, $\delta=1$ if $b_{j} \equiv 0(\bmod g)(j=1,2, \ldots, s)$ and $\delta=0$ otherwise, $S(f H, q, B, g)$ is the Gaussian sum of certain type. He proved that if $f$ is an integral positive quadratic form with $s$ variables, $s>4$, then the Fourier coefficients of the Eisenstein series are the Hardy-Littlewood singular series that corresponds to the system of equations

$$
f\left(x_{1}, x_{2}, \ldots, x_{s}\right)=n \quad x \equiv B(\bmod g) .
$$

Here $n$ is a natural number and $x$ is a variable column vector with $s$ components. Vepkhvadze [140] proved that the function

$$
=\delta+\frac{e^{(2 m-s) \pi i / 4}}{g^{s / 2}|d|^{1 / 2}} \sum_{q=1}^{\infty} \sum_{\substack{H=-\infty \\(H, q)=1}}^{\infty} \frac{E(\tau, z ; f, B, g)}{q^{s / 2}(q \tau-H)^{m / 2}(q \bar{\tau}-H)^{(s-m) / 2}|q \tau-H|^{z}}
$$

regular for any fixed $\tau$ with $\operatorname{Im} \tau>0$ and $\operatorname{Re} z>2-\frac{s}{2}$, can be analytically extended in a neighborhood of $z=0$ when $f$ is any integral quadratic form with $s \geq 2$ variables (exceptions are zero forms with $s=2$ or 3 variables and zero forms with $s=4$ variables whose discriminant is a perfect square) and when $f\left(b_{1}, \ldots, b_{s}\right) \equiv 0(\bmod g)$. In addition, for positive quadratic forms he derived formulas for computing the Fourier coefficients of the series $\left.E(\tau, z ; f, B, g)\right|_{z=0}$.

5.1. Hecke [34] constructed a basis of a space of second order spherical functions with respect to a positive integral quadratic form with an even number of variables, and Lomadze [72] constructed a basis of a space of fourth order spherical functions with respect to the above-mentioned form. Lomadze ([75], [79]) used this result to construct a basis of a space of cusp forms of type $(-6, q, 1)$ when $q=3,5,7,11$, and the basis of a space of cusp forms of type 
$(-6, q, \chi)$ when $q=5,13,17$ in the form of generalized four-fold theta-series with spherical functions. Kachakhidze [38] constructed a basis of a space of cusp forms of type $(-6, q, 1)$ when $q=13,17$. Using Hecke's basis of a space of second order spherical functions constructed again in terms of generalized fourfold theta-series with spherical functions, Gongadze [31] constructed a basis of a space of cusp forms of type $(-4, q, 1)$ when $q=5,7,11$ and Shavgulidze[106] when $q=13,19,23$. Shavgulidze [107] also constructed a basis of a space of cusp forms of type $(-4, q, \chi)$ when $q=13,29$. It should be remarked here that the construction of the latter basis required the finding of a reduced positive quaternary quadratic form with discriminant $q^{2}$. Shavgulidze proposed quite a simple method of finding such a form when $q$ is an arbitrary odd prime number. Until then such quadratic forms had been known only when $q=3,5,7$ (Germann [16]), $q=11$ (Hecke [34]) and $q=17$ (Hermann [35]).

Lomadze, Gongadze, Shavgulidze and Kachakhidze also showed that the constructed bases of spaces of cusp forms can be used to derive exact formulas for the number of representations of a natural number by quadratic forms of the respective type. Later Lomadze ([74], [77], [80]) constructed the bases of spaces of spherical functions of arbitrary order with respect to the quadratic forms mentioned above and used these bases for constructing as generalized four-fold theta-series the bases of spaces of cusp forms

of type $(-8, q, 1)$ for $q=3,5,7$,

of type $(-8, q, \chi)$ for $q=5,13$,

of type $(-10, q, 1)$ for $q=3,5$, and

of types $(-10,5, \chi)$ and $(-12,5, \chi)$.

5.2. Let $F_{1}=x_{1}^{2}+x_{1} x_{2}+a x_{2}^{2}$, where $a$ is a given natural number, be the quadratic form with a negative odd discriminant $q$, and $F_{k}$ be the direct sum of $k$ quadratic forms $F_{1}$. Denote by $r\left(n ; F_{k}\right)$ the number of representations of a natural number $n$ by the quadratic form $F_{k}$. For the first time ever Petersson [103] obtained exact formulas for the function $r\left(n ; F_{2}\right)$ when $a=1,2,3,6$, i.e., when $q=-3,-7,-11,-23$, respectively; for the function $r\left(n ; F_{3}\right)$ when $a=$ 1,2; for the function $r\left(n ; F_{k}\right)$ when $k=4,5,6$ and $a=1$. Lomadze ([82], [81], [83]) obtained exact formulas for the function $r\left(n ; F_{k}\right)$ for all $2 \leq k \leq 20$ when $F_{1}=x_{1}^{2}+x_{1} x_{2}+x_{2}^{2}$, and for all $2 \leq k \leq 11$ when $F_{1}=x_{1}^{2}+x_{1} x_{2}+2 x_{2}^{2}$. To this end, for all $k$ individually he constructed the bases of spaces of cusp forms $S_{k}\left(\Gamma_{0}(3), \chi^{k}\right)$ and $S_{k}\left(\Gamma_{0}(7), \chi^{k}\right)$ in terms of generalized multiple thetaseries with spherical functions. His tools were the standard spherical functions discussed in Subsection 5.1, also Hecke's results on cusp forms and Eisenstein series. The obtained formulas like the formulas for the function $r_{2 k}(n)$ from Section 1, obey a certain law.

Kachakhidze [39] succeeded in obtaining for the function $r\left(n ; F_{k}\right)$ somewhat different exact formulas for all $k, 6 \leq k \leq 17$, when $F_{1}=x_{1}^{2}+x_{1} x_{2}+x_{2}^{2}$. He constructed not one basis but a whole system of bases of the space $S_{k}\left(\Gamma_{0}(3), \chi^{k}\right)$ for $k \geq 6$. He also constructed a system of bases of the space $S_{k}\left(\Gamma(7), \chi^{k}\right)$ for $k \geq 3$ and he obtained formulas for the function $r\left(n ; F_{k}\right)$ for all $3 \leq k \leq 11$ when 
$F_{1}=x_{1}^{2}+x_{1} x_{2}+2 x_{2}^{2}$. Further, he constructed a basis of the space $S_{k}\left(\Gamma(11), \chi^{k}\right)$ for any integer $k \geq 3$ and derived formulas for the function $r\left(n ; F_{k}\right)$ for all $3 \leq k \leq 8$ when $F_{1}=x_{1}^{2}+x_{1} x_{2}+3 x_{2}^{2}$. The formulas obtained by Kachakhidze obey a certain law.

Let now $\Phi_{2}$ be the integral positive reduced quadratic form whose discriminant is $d=5$ or 13 , and $\Phi_{2 m}$ be the direct sum of $m$ quadratic forms $\Phi_{2}$. Kachakhidze [40] constructed the bases of spaces of cusp forms $S_{2 m}\left(\Gamma_{0}(d), \chi^{m}\right)$ for $m \geq 2$ and use these bases to obtain exact formulas for the function $r\left(n ; \Phi_{2 m}\right)$ for all $m, 2 \leq m \leq 5$, when $d=5$, and for all $m, 2 \leq m \leq 4$, when $d=13$.

6.1. As has been mentioned above, mathematicians searched for the additional terms of exact formulas for the number of representations of a natural number by a positive integral quadratic form that have a simple arithmetic meaning among the products of Jacobi theta-series or among the products of theta-series with characteristics and their derivatives or among the Fourier coefficients of generalized multiple theta-series with spherical functions.

Kloosterman [42] generalized an ordinary theta-function with characteristics. The next generalization of this function is due to Vepkhvadze ([141], [143]). Let $f$ be an integral positive quadratic form with $s$ variables, $A$ be its matrix and $N$ be its level, $X$ be a variable column vector with $s$ components, $\tau$ be a complex variable ( $\operatorname{Im} \tau>0), z$ be a column vector with $s$ complex components, $g$ and $h$ be special vectors with respect to the matrix $A$ and with integral components for which $A g \equiv 0(\bmod N)$ and $A h \equiv 0(\bmod N), P_{\nu}(X)$ be a spherical function of order $\nu$ with respect to the quadratic form $f$. Then the function

$$
\vartheta_{g h}\left(z \mid \tau ; P_{\nu}, f\right)=\sum_{X \equiv g(\bmod N)}(-1)^{\frac{h^{T} A(X-g)}{N^{2}}} P_{\nu}(X) e^{\frac{\pi i \tau X^{T} A X}{N^{2}}} e^{\frac{2 \pi i X^{T} A z}{N}}
$$

is a generalized $s$-fold theta-series with characteristics and spherical functions. Vepkhvadze established the basic properties of this function and studied its behavior with respect to the subgroup of linear permutations $\Gamma_{0}(N)$. He proved the theorem which gives a necessary and sufficient condition for a certain linear combination of such theta-functions to be an entire modular form of the type $\left(-\left(\frac{s}{2}+\nu\right), N, v(L)\right)$, where $\nu$ is the order of a spherical function with respect to a positive integral quadratic form with $s \geq 2$ variables, $N$ is the level of this quadratic form, and $v(L)$ is a system of multipliers with respect to the group $\Gamma_{0}(N)$ ( $L$ is any permutation matrix of $\Gamma_{0}(N)$ ). As has already been said in Section 3, the sufficient condition of Vepkhvadze's theorem had been obtained by Lomadze previously only for a few particular cases and only for an ordinary theta-function with characteristics. Vepkhvadze ([142], [144]) used his theorem to derive exact formulas for the number of representations of a natural number by quadratic forms of the type

$$
f=\sum_{i=1}^{s_{1}} x_{i}^{2}+2 \sum_{i=s_{1}+1}^{s_{2}} x_{i}^{2}+4 \sum_{i=s_{2}+1}^{5} x_{i}^{2}
$$


with five variables when $1 \leq s_{1} \leq 4$ and $1 \leq s_{2} \leq 4$, and by quadratic forms of the type

$$
f=\sum_{i=1}^{s} x_{i}^{2}+2 \sum_{i=s+1}^{7} x_{i}^{2}
$$

with seven variables when $1 \leq s \leq 6$, which belong to multi-class genera. The additional terms of the obtained formulas are the sums extended to certain representations of integers by positive diagonal ternary quadratic forms which belong to one-class genera.

Using the generalized theta-functions with characteristics and spherical functions introduced by Vepkhvadze, Tsalugelashvili [123] constructed the bases of spaces of cusp forms corresponding to positive diagonal quadratic forms of level 8 and with an even number of variables, i.e., to quadratic forms of the type

$$
f=\sum_{i=1}^{k} x_{i}^{2}+2 \sum_{i=k+1}^{s} x_{i}^{2}, \quad 2 \mid s .
$$

Vepkhvadze and Tsulugelashvili [145] used these bases to obtain exact formulas for the number of representations of a natural number by all such forms when $s=10$.

6.2. Lomadze ([71], [86], [88]) studied the behavior of $n$-th order derivatives of ordinary theta-functions with characteristics with respect to the group $\Gamma_{0}(4 N)$. He proved that if certain conditions are satisfied, then for a given natural number $N$ the functions

$$
\begin{aligned}
& \text { (i) } \frac{1}{N_{1}} \vartheta_{g_{1} h_{1}}^{\prime \prime \prime}\left(\tau ; 0,2 N_{1}\right) \vartheta_{g_{2} h_{2}}^{\prime}\left(\tau ; 0,2 N_{2}\right)-\frac{1}{N_{2}} \vartheta_{g_{2} h_{2}}^{\prime \prime \prime}\left(\tau ; 0,2 N_{2}\right) \vartheta_{g_{1} h_{1}}^{\prime}\left(\tau ; 0,2 N_{1}\right) \text {, } \\
& \text { (ii) } \frac{1}{N_{1}^{2}} \vartheta_{g_{1} h_{1}}^{(4)}\left(\tau ; 0,2 N_{1}\right) \vartheta_{g_{2} h_{2}}\left(\tau ; 0,2 N_{2}\right)+\frac{1}{N_{2}^{2}} \vartheta_{g_{2} h_{2}}^{(4)}\left(\tau ; 0,2 N_{2}\right) \vartheta_{g_{1} h_{1}}\left(\tau ; 0,2 N_{1}\right) \\
& -\frac{6}{N_{1} N_{2}} \vartheta_{g_{1} h_{1}}^{\prime \prime}\left(\tau ; 0,2 N_{1}\right) \vartheta_{g_{2} h_{2}}^{\prime \prime}\left(\tau ; 0,2 N_{2}\right), \\
& \text { (iii) }\left\{\frac{1}{N_{1}} \vartheta_{g_{1} h_{1}}^{\prime \prime}\left(\tau ; 0,2 N_{1}\right) \vartheta_{g_{2} h_{2}}\left(\tau ; 0,2 N_{2}\right)-\frac{1}{N_{2}} \vartheta_{g_{2} h_{2}}^{\prime \prime}\left(\tau ; 0,2 N_{2}\right) \vartheta_{g_{1} h_{1}}\left(\tau ; 0,2 N_{1}\right)\right\} \\
& \times \vartheta_{g_{3} h_{3}}^{\prime}\left(\tau ; 0,2 N_{3}\right) \vartheta_{g_{4} h_{4}}\left(\tau ; 0,2 N_{4}\right), \\
& \text { (iv) } \prod_{k=1}^{3} \vartheta_{g_{k} h_{k}}^{\prime}\left(\tau ; 0,2 N_{k}\right) \vartheta_{g_{4} h_{4}}\left(\tau ; 0,2 N_{4}\right)
\end{aligned}
$$

are cusp forms with respect to the group $\Gamma_{0}(4 N)$ which are of weight 5 and have the character $\chi(\delta)=\operatorname{sgn} \delta\left(\frac{-\Delta}{|\delta|}\right)$ ( $\Delta$ is the determinant of a positive integral quadratic form with ten variables for which these functions are used). He constructed similar cusp forms with respect to the group $\Gamma_{0}(4 N)$ whose weight is 6 and the character is $\chi(\delta)=\left(\frac{\Delta}{|\delta|}\right)$ (here $\Delta$ is the determinant of a positive integral quadratic form with twelve variables for which these functions are used). These cusp forms enable one to obtain exact formulas for the number of representations of a natural number by some integral positive quadratic forms 
with ten and twelve variables, whose additional terms have a sufficiently simple arithmetic meaning. For the sake of simplicity, among quadratic forms with ten variables of type (4), Lomadze chose for consideration a primitive quadratic form of the type

$$
f=\left\{a_{1}, a_{1}, a_{2}, a_{2}, a_{3}, a_{3}, a_{4}, a_{4}, a_{5}, a_{5}\right\}
$$

and derived convenient formula for computing the sums of the singular series that corresponds to this quadratic form [89]. He and Daneliya [98] further obtained exact formulas for the number of representations of a natural number by fourteen such quadratic forms whose coefficients are 1 and 2 or 1 and 4 or 1,2 , and 4 . To this end, among the four functions presented above they chose, for each concrete quadratic form, additional functions and proved identities of type (3).

Lomadze [91] derived exact formulas for the number of representations of a natural number by the quadratic forms $F_{3}, F_{2} \oplus \Phi_{1}, F_{1} \oplus \Phi_{2}$ and $\Phi_{3}$ which are the direct sums of binary reduced quadratic forms of discriminant 23

$$
F_{1}=x_{1}^{2}+x_{1} x_{2}+6 x_{2}^{2} \text { and } \Phi_{1}=2 x_{1}^{2}+x_{1} x_{2}+3 x_{2}^{2} .
$$

He [94] also derived explicit exact formulas for the number of representations of a natural number by three quadratic forms which are the direct sums of quaternary reduced quadratic forms of discriminant $19^{2}$. The additional terms of these formulas have a simple arithmetic meaning.

Lomadze ([92], [93], [95], [96]) proposed a method of obtaining explicit exact formulas for the number of representations of a natural number by positive diagonal integral quadratic forms with 12 and 14 variables when the pairwise adjacent coefficients are equal.

\section{REFERENCES}

1. R. I. BERIDze, On the summation of the singular series of Hardy-Littlewood. (Russian) Soobshch. Akad. Nauk Gruzin. SSR 38(1965), 529-534.

2. R. I. BERIDze, The representation of numbers by certain quadratic forms in four variables. (Russian) Soobshch. Akad. Nauk Gruzin. SSR 50(1968), 267-272.

3. R. I. BERIDZE, The representation of numbers by certain pairs of binary quadratic forms. (Russian) Trudy Gruzin. Politekh. Inst. 1968, No. 2 (122), 7-15.

4. R. I. BERIDzE, The representation of numbers by certain quadratic forms in eight variables. (Russian) Trudy Tbiliss. Gos. Univ. A 1 (137) (1971), 5-16.

5. R. I. BERIDZE, On the representation of numbers by some quadratic forms with eight variables. (Russian) Trudy Tbiliss. Univ. 314, Mat. Mekh. Astronom. No. 29 (1993), $5-14$.

6. R. I. Beridze and G. P. Gogishvili, The number of representations of numbers by certain quadratic forms in six variables. (Russian) Trudy Tbiliss. Mat. Inst. Razmadze $\mathbf{5 7}(1977), 5-15$.

7. H. Bessel, Elementar-arithmetische Untersuchungen über die Darstellung einer natürlichen Zahl als Summe von Quadratzahlen. Diss. Kénigsberg. 45. S. Borna, Noske, 1929.

8. F. VAN DER BliJ, On the theory of quadratic forms. Ann. of Math. (2) 50(1949), $875-883$. 
9. F. VAN DER BliJ, Binary quadratic forms of discriminant -23. Nederl. Akad. Wetensch. Proc. Ser. A. 55 = Indagationes Math. 14(1952) 498-503.

10. V. V. Bulygin, Sur une application des fonctions elliptiques au probléme de représentation des nombres entiers par une somme de carrés. St. Petersbourg, Bull. Acad. Sci. (6) 8(1914), 389-404.

11. L. E. Dickson, Modern elementary theory of numbers. University of Chicago Press, Chicago, 1939.

12. P. G. L. Dirichlet, Recherches sur diverses applications de l'Analyse infinitésimale à la Théorie des Nombres. J. Reine Angew. Math. (1839), 324-369.

13. T. Estermann, On the representations of a number as a sum of squares. Acta Arith., Warszawa, 2(1936), 47-79.

14. T. Estermann, On the representations of a number as a sum of three squares. Proc. London Math. Soc. (3) 9(1959), 575-594.

15. M. S. Gelashvili, The representation of numbers by certain quadratic forms in four variables. (Russian) Trudy Tbiliss. Univ. A 1 (137)(1971), 25-34.

16. K. Germann, Tabellen reduzierter, positiver quaternärer quadratischer Formen. Comment. Math. Helv. 38(1963), 56-83.

17. G. P. Gogishvili, The representation of numbers by quaternary quadratic forms with coefficients equal to 1 and 11. (Russian) Soobshch. Akad. Nauk Gruzin. SSR 49(1968), No. 1, 9-12.

18. G. P. Gogishvili, number of representations of numbers by certain quaternary quadratic forms. (Russian) Soobshch. Akad. Nauk Gruzin. SSR 56(1969), No. 3, 525-528.

19. G. P. Gogishvili, The summation of a singular series that is connected with diagonal quadratic forms with four variables. (Russian) Trudy Tbiliss. Mat. Inst. Razmadze 38(1970), 5-30.

20. G. P. Gogishvili, number of representations of numbers by positive quaternary diagonal quadratic forms. (Russian) Soobshch. Akad. Nauk Gruzin. SSR 59(1970), 537-540.

21. G. P. Gogishvili, The number of representations of numbers by positive quaternary diagonal quadratic forms. (Russian) Trudy Tbiliss. Mat. Inst. Razmadze 40(1971), 59105.

22. G. P. Gogishvili, The finiteness of the number of definite classes of positive primitive quadratic forms. (Russian) Trudy Tbiliss. Mat. Inst. Razmadze 45(1974), 78-110.

23. G. P. Gogishvili, One-class genera of certain ternary quadratic forms. (Russian) Soobshch. Akad. Nauk Gruzin. SSR 81(1976), No. 2, 313-315.

24. G. P. Gogishvili, A relation between the number of representation of numbers by quadratic forms and the corresponding singular series. (Russian) Trudy Tbiliss. Mat. Inst. Razmadze 57(1977), 40-62.

25. G. P. Gogishvili, A type of formula for the number of representations of numbers by positive quadratic forms. Trudy Tbiliss. Mat. Inst. Razmadze 63(1980), 25-35.

26. G. P. Gogishvili, On the representation of numbers by positive binary quadratic forms. (Russian) Soobshch. Akad. Nauk Gruzin. SSR 107(1982), No. 2, 257-260.

27. G. Gogishvili, On the general unimprovable estimates of the singular series of positive quadratic forms. Analytic and probabilistic methods in number theory. Proceedings of the 2nd international conference in honour of J. Kubilius, Palanga, Lithuania, September 23-27, 1996. New trends in probability and statistics, Vol. 4, 35-43, VSP, Utrecht, 1997.

28. R. Sh. Gongadze, On the representation of numbers by certain quadratic forms of four variables. (Russian) Soobshch. Akad. Nauk Gruzin. SSR 28(1962), 385-392. 
29. R. Sh. Gongadze, Representation of numbers by the forms $x^{2}+3 y^{2}+4 z^{2}+12 t^{2}$ and $x^{2}+2 y^{2}+32 z^{2}+32 t^{2}$. (Russian) Soobshch. Akad. Nauk Gruzin. SSR 46(1967), 33-40.

30. R. Sh. Gongadze, The representation of numbers by certain forms of the type $x^{2}+$ $2^{2 k+1} y^{2}+32 z^{2}+32 t^{2}$. (Russian) Soobshch. Akad. Nauk Gruzin. SSR 50(1968), 519-524.

31. R. Sh. Gongadze, On the representation of numbers by quadratic forms of the type (-4, q, 1). (Russian) Trudy Tbiliss. Univ. 176, Mat. Mekh. Astronom. (1976), 15-27.

32. G. H. HARDY, On the representation of a number as the sum of any number of squares, and in particular of five or seven. Nat. Acad. Proc. 4(1918), 189-193.

33. G. H. HARDY, On the representation of a number as the sum of any number of squares, and in particular of five. American M. S. Trans. 21(1920), 255-284.

34. E. Hecke, Analytische Arithmetik der positiven quadratischen Formen. Danske Vid. Selsk. Math.-Fys. Medd. 17(1940), No. 12, 134 pp.

35. O. Herrmann, Kongruenzeigenschaften der Partitionenfunktion. J. Number Theory 1(1969), 431-458.

36. C. C. J. JACOBI, Theorie der elliptischen Functionen aus den Eigenschaften der Thetareihen abgeraitet. Gesammelte Werke, herausgegeben von Borchardt, B. I., Berlin, 1881, 497-538.

37. B. W. Jones and G. PALL, Regular and semiregular positive ternary quadratic forms. Acta Math., Uppsala, 70(1939), 165-191.

38. N. D. Kachakhidze, Parabolic and quadratic forms of type $(-6,13,1)$ and $(-6,17,1)$. (Russian) Trudy Tbiliss. Univ. 278, Mat. Mekh. Astronom. No. 24 (1988), 66-91.

39. N. KACHAKHIDZE, On the representation of numbers by the direct sums of some binary quadratic forms. Georgian Math. J. 5(1998), No. 1, 55-70.

40. N. KACHAKHIDZE, On the representation of numbers by the direct sums of some quaternary quadratic forms. Georgian Math. J. 8(2001), No. 1, 87-95.

41. H. D. Kloosterman, On the representation of numbers in the form $a x^{2}+b y^{2}+c z^{2}+$ $d t^{2}$. Proceedings L. M. S. (2) 25(1926), 143-173.

42. H. D. Kloosterman, The behaviour of general theta functions under the modular group and the characters of binary modular congruence groups. I. Ann. of Math. (2) 47(1946), 317-375.

43. J. Liouville, Sur la forme $x^{2}+y^{2}+z^{2}+5 t^{2}$. J. Math. Pures Appl. (2) 9(1864), 1-12.

44. J. Liouville, Sur la forme $x^{2}+5\left(y^{2}+z^{2}+t^{2}\right)$. ibid., 17-22.

45. J. Liouville, Extrait d'uno Lettre adresséo à U. Besge. ibid., 296-298.

46. J. Liouville, Sur la forme $x^{2}+2 y^{2}+3 z^{2}+6 t^{2}$. ibid., 299-312.

47. J. Liouville, Sur la forme $x^{2}+y^{2}+5 z^{2}+5 t^{2}$. J. Math. Pures Appl. (2) 10(1865), $1-8$.

48. J. Liouville, Sur la forme $x^{2}+y^{2}+9 z^{2}+9 t^{2}$. ibid., $14-20$.

49. J. Liouville, Sur la forme $x^{2}+y^{2}+4\left(z^{2}+t^{2}+u^{2}+v^{2}\right)$. ibid., 71-72.

50. J. Liouville, Sur la forme $x^{2}+y^{2}+2\left(z^{2}+t^{2}\right)+4\left(u^{2}+v^{2}\right)$. ibid., 145-150.

51. J. Liouville, Sur la forme $x^{2}+y^{2}+z^{2}+t^{2}+4 u^{2}+4 v^{2}$. ibid., 161-168.

52. J. Liouville, Sur la formes $x^{2}+y^{2}+6 z^{2}+6 t^{2}, 2 x^{2}+2 y^{2}+3 z^{2}+3 t^{2}$. ibid., 359-360.

53. G. A. Lomadze, On the representation of numbers by sums of squares. (Russian) Trudy Tbiliss. Mat. Inst. Razmadze 16(1948), 231-275.

54. G. A. Lomadze, On the representation of numbers by sums of an odd number of squares. (Georgian) Trudy Tbiliss. Mat. Inst. Razmadze 17(1949), 281-314.

55. G. A. Lomadze, On the representation of numbers by sums of squares. (Russian) Trudy Tbiliss. Mat. Inst. Razmadze 20(1954), 47-87. 
56. G. Lomadze, Über die Darstellung der Zahlen durch einige quaternáre quadratische Formen. Acta Arith. 5(1959), 125-170.

57. G. A. Lomadze, On the representation of numbers by some quadratic forms in four variables. (Russian) Trudy Tbiliss. Univ. 76(1959), 107-159.

58. G. A. LOMADZE, On the representation of numbers by some ternary quadratic forms. (Russian) Trudy Tbiliss. Mat. Inst. Razmadze 27(1960), 115-141.

59. G. Lomadze, Über die Darstellung der Zahlen durch einige ternáre quadratische Formen. Acta Arith. 6(1960/1961), 225-275.

60. G. A. Lomadze, On the representation of numbers by binary quadratic forms. (Russian) Trudy Tbiliss. Univ. 84 Mat. Mekh. (1962), 285-290.

61. G. A. Lomadze, The scientific work of Arnold Walfisz. Acta Arith. 10(1964), 227-237.

62. G. A. Lomadze, On the representation of numbers by cetain quaternary quadratic forms. (Russian) Trudy Tbiliss. Univ. 110, Mat. Mekh. Astronom. IV (1965), 163-180.

63. G. A. Lomadze, On the representation of numbers by positive binary diagonal quadratic forms. (Russian) Mat. Sb. (N.S.) 68 (110)(1965), 282-312.

64. G. A. Lomadze, Representation of numbers by certain quadratic forms in six variables. I. (Russian) Trudy Tbiliss. Univ. 117 Mat. Mekh. Astronom. V (1966), 7-43.

65. G. A. Lomadze, Representation of numbers by certain quadratic forms in six variables. II. (Russian) Trudy Tbiliss. Univ. 129, Mat. Mekh. Astronom. VI (1968), 275-297.

66. G. A. Lomadze, The number of representations of numbers by certain quadratic forms in four variables. (Russian) Soobshch. Akad. Nauk Gruzin. SSR 50(1968), 295-300.

67. G. A. Lomadze, The number of representations of numbers by the forms $x_{1}^{2}+3 x_{2}^{2}+$ $36 x_{3}^{2}$ and $x_{1}^{2}+12 x_{2}^{2}+36 x_{3}^{2}$. (Russian) Soobshch. Akad. Nauk Gruzin. SSR 51(1968), 25-30.

68. G. A. Lomadze, A representation of numbers by positive ternary diagonal quadratic forms. I, II. (Russian) Acta Arith. 19(1971), 267-305; 19(1971), 387-407.

69. G. A. Lomadze, The number of representations of numbers by quadratic forms with four variables. (Russian) Trudy Tbiliss. Mat. Inst. Razmadze 40(1971), 106-139.

70. G. A. Lomadze, Formulae for the number of representations of numbers by all primitive positive ternary diagonal quadratic forms that belong to one-class genera. (Russian) Trudy Tbiliss. Mat. Inst. Razmadze 40(1971), 140-179.

71. G. A. Lomadze, The behavior of the derivatives of theta-functions under linear substitutions. (Russian) Trudy Tbiliss. Univ. A 4 (146)(1972), 15-27.

72. G. A. Lomadze, The basis of the space of spherical functions of order four with respect to a positive quadratic form. (Russian) Soobshch. Akad Nauk Gruzin. SSR 69(1973), $533-536$.

73. G. A. Lomadze, The number of representations of numbers by positive quadratic forms in six variables. (Russian) Trudy Tbiliss. Mat. Inst. Razmadze 45(1974), 111133.

74. G. A. Lomadze, The basis of the space of spherical functions with respect to a positive quadratic form. (Russian) Collection of articles on number theory, 2. Trudy Tbiliss. Mat. Inst. Razmadze 45(1974), 134-145.

75. G. A. Lomadze, Cusp forms of prime level and of Haupttypus. I. (Russian) Trudy Tbiliss. Mat. Inst. Razmadze 45(1974), 146-161.

76. G. A. Lomadze, On representation of numbers by sum of $8 t+1$ squares. (Russian) Trudy Tbiliss. Univ. 179(1976), 63-66.

77. G. A. Lomadze, Cusp forms of prime level and of Haupttypus. II. (Russian) Trudy Tbiliss. Mat. Inst. Razmadze 57(1977), 63-81. 
78. G. A. Lomadze, Formulas of the number of representations of numbers by certain regular and semiregular ternary quadratic forms belonging to two-class genera. (Russian) Acta Arith. 34(1977/78), No. 2, 131-162.

79. G. A. Lomadze, Cusp forms of prime level of nebentypus. I. (Russian) Trudy Tbiliss. Mat. Inst. Razmadze 63(1980), 36-53.

80. G. A. Lomadze, Cusp forms of prime level of Nebentypus. II. (Russian) Trudy Tbiliss. Mat. Inst. Razmadze 72(1983), 40-54.

81. G. A. Lomadze, Representation of numbers by the direct sum of quadratic forms of type $x_{1}^{2}+x_{1} x_{2}+x_{2}^{2}$. (Russian) Trudy Tbiliss. Univ. 288, Mat. Mekh. Astronom. No. 26 (1989), 5-21.

82. G. A. Lomadze, Representation of numbers by sums of the quadratic forms $x_{1}^{2}+$ $x_{1} x_{2}+x_{2}^{2}$. (Russian) Acta Arith. 54(1989), No. 1, 9-36.

83. G. A. Lomadze, On the number of representations of numbers by the direct sum of quadratic forms of type $x_{1}^{2}+x_{1} x_{2}+2 x_{2}^{2}$. (Russian) Trudy Tbiliss. Univ. 299, Mat. Mekh. Astronom. No. 27 (1990), 12-59 (1991).

84. G. A. Lomadze, Some arithmetic applications of the theory of modular forms. (Russian) Trudy Mat. Inst. Steklov. 200(1991), 236-244; English transl.: Proc. Steklov Inst. Math. 1993, No. 2 (200), 261-270.

85. G. Lomadze, On some entire modular forms of half integral weight on the group $\Gamma_{0}(4 N)$. Analytic and probabilistic methods in number theory. Proc. Int. Conf. in Honour of J. Kubilius, Palanga/Lith. 1991, New trends in probability and statistics, 2(1992), $57-67$.

86. G. Lomadze, On some entire modular forms of weights 5 and 6 for the congruence group $\Gamma_{0}(4 N)$. Georgian Math. J. 1(1994), No. 1, 53-76.

87. G. A. Lomadze, On the number of representations of natural numbers by sums of nine squares. (Russian) Acta Arith. 68(1994), No. 3, 245-253.

88. G. Lomadze, Construction of entire modular forms of weights 5 and 6 for the congruence group $\Gamma_{0}(4 N)$. Georgian Math. J. 2(1995), No. 2, 189-199.

89. G. Lomadze, On the number of representations of integers by some quadratic forms in ten variables. Georgian Math. J. 2(1995), No. 5, 491-516.

90. G. Lomadze, On some entire modular forms of weights $7 / 2$ and $9 / 2$ for the congruence group $\Gamma_{0}(4 N)$. Georgian Math. J. 3(1996), No. 5, 485-500.

91. G. Lomadze, On the number of representations of positive integers by a direct sum of binary quadratic forms with discriminant -23. Georgian Math. J. 4(1997), No. 6, $523-532$.

92. G. Lomadze, Summation of singular series corresponding to representations of numbers by some quadratic forms in twelve variables. Georgian Math. J. 5(1998), No. 4, 367-384.

93. G. Lomadze, On the number of representations of integers by quadratic forms in twelve variables. Georgian Math. J. 5(1998), No. 6, 545-564.

94. G. Lomadze, On the number of representations of positive integers by a direct sum of quaternary quadratic forms with discriminant $19^{2}$. Proc. A. Razmadze Math. Inst. 123(2000), 53-59.

95. G. Lomadze, Summation of singular series corresponding to the representation of integers by some quadratic forms in fourteen variables. Georgian Math. J. 7(2000), No. $2,355-372$.

96. G. LOMADZE, On the number of representations of positive integers by some quadratic forms in fourteen variables. Georgian Math. J. 7(2000), No. 3, 551-564. 
97. G. Lomadze, On the number of representations of positive integers by the quadratic form $x_{1}^{2}+\cdots+x_{8}^{2}+4 x_{9}^{2}$. Georgian Math. J. 8(2001), No. 1, 111-127.

98. G. A. Lomadze and A. N. DANEliYA, On the number of representations of integers by some quadratic forms in 10 variables. (Russian) Trudy. Tbiliss. Univ. 324, Mat. Mekh. Astronom. No. 31 (1997), 16-46.

99. H. MAAss, Konstruktion ganzer Modulformen halbzahliger Dimension mit $\vartheta$-Multiplikatoren in einer und zwei Variablen. Abh. math. Sem. Hansische Univ. 12(1938), 133-162.

100. A. V. Malyshev, On the representation of integers by positive quadratic forms. (Russian) Trudy Mat. Inst. Steklov 65(1962), 212 pp.

101. L. J. Mordell, On the representations of numbers as a sum of $2^{r}$ squares. Quart. J. 48(1918), 93-104.

102. L. J. Mordell, On the representations of a number as a sum of an odd number of squares. Cambr. Phil. Trans. 22(1919), 361-372.

103. H. Petersson, Modulfunktionen und quadratische Formen. Ergebnisse der Mathematik und ihrer Grenzgebiete [Results in Mathematics and Related Areas], 100. Springer-Verlag, Berlin, 1982.

104. K. G. Ramanathan, On the analytic theory of quadratic forms. Acta Arith. 21(1972), 423-436.

105. R. A. Rankin, Sums of squares and cusp forms. Amer. J. Math. 87(1965), No. 4, $857-860$.

106. K. Sh. Shavgulidze, Cusp and quadratic forms of type $(-4, q, 1)$. (Russian) Trudy Tbiliss. Univ. 214, Mat. Mekh. Astronom. No. 9 (1980), 194-219.

107. K. Sh. Shavgulidze, Cusp and quadratic forms of type $(-4, q, \chi)$. (Russian) Trudy Tbiliss. Univ. 215, Mat. Mekh. Astronom. No. 10 (1980), 95-116.

108. C. L. Siegel, Über die analytische Theorie der quadratischen Formen. Ann. of Math. (2) 36(1935), No. 3, 527-606.

109. C. L. Siegel, Gesammelte Abhandlungen. Band IV. [Collected papers. Vol. IV] Springer-Verlag, Berlin-New York, 1979.

110. CH. J. S. Smith, Memoir on the theta and omega functions. Collected Methematical Papers, ed. by Gliasher, v. II, 415-622. Oxford, 1894.

111. H. Streefkerk, Over het Aantal Oplossingen der Diophantische Vergelijking $U=$ $\sum_{i=1}^{s}\left(A x_{i}^{2}+B x_{i}+C\right)$. (Dutch) [On the Number of Solutions of the Diophantine Equation $\left.U=\sum_{i=1}^{s}\left(A x_{i}^{2}+B x_{i}+C\right)\right]$ Thesis, Free University of Amsterdam, 1943.

112. Z. SuetunA, Über die Maximalordnung einiger Funktionen in der Idealtheorie. II. Journal Faculty of Science Tokyo 1(1926), 249-283.

113. Z. Suetuna, Über die Maximalordnung einiger Funktionen in der Idealtheorie. III. Journal Faculty of Science Tokyo 1(1926), 349-371.

114. L. A. Sulakvelidze, The representation of numbers by certain ternary quadratic forms that belong to multiclass genera. (Russian) Trudy Tbiliss. Univ. A 8 (153)(1974), 39-56.

115. L. A. Sulakvelidze, Representation of numbers by positive ternary quadratic forms. I. (Russian) Trudy Tbiliss. Univ. 185(1977), 5-42.

116. L. A. Sulakvelidze, Representation of numbers by positive ternary quadratic forms. II. (Russian) Trudy Tbiliss. Univ. 189(1977), 29-52.

117. L. A. Sulakvelidze, The representation of numbers by certain positive ternary quadratic forms. (Russian) Soobshch. Akad. Nauk Gruzin. SSR 88(1977), No. 1, 21-24. 
118. L. A. Sulakvelidze, The number of representations of numbers by certain regular and semiregular ternary quadratic forms belonging to multiclass genera. (Russian) Trudy Tbiliss. Mat. Inst. Razmadze 63(1980), 54-69.

119. L. A. Sulakvelidze, Representations of numbers by positive ternary quadratic forms. III. (Russian) Trudy Tbiliss. Univ. 214, Mat. Mekh. Astronom. No. 9 (1980), 146-193.

120. L. A. Sulakvelidze, The number of representations of numbers by certain regular and semiregular ternary quadratic forms belonging to multiclass genera. II. (Russian) Trudy Tbiliss. Mat. Inst. Razmadze 72(1983), 55-67.

121. L. A. Sulakvelidze, Some formulas of Liouville type for the number of representations of numbers by positive quadratic forms with six variables. (Russian) Trudy Tbiliss. Mat. Inst. Razmadze 84(1987), 55-65.

122. L. A. Sulakvelidze, On the universality of genera of positive ternary quadratic forms. (Russian) Proc. A. Razmadze Math. Inst. 109(1995), 67-73.

123. N. Tsalugelashvili, The construction of the bases of some eight step cusp form spaces. Bull. Georgian Acad. Sci. 157(1998), No. 3, 359-361.

124. N. Tsalugelashvili and T. Vepkhvadze, The construction of the bases of some eight level cusp form spaces. Georgian Math. J. 7(2000), No. 4, 793-798.

125. J. V. UspenskiJ, Über die Anzahl der Darstellungen der Zahlen durch gewisse quadratische Formen mit 4 und 6 Variabeln. (Russian) Comm. Soc. Math. (2) 15(19151917), 81-147.

126. J. V. UspenskiJ, On the number of representations of integers by certain ternary quadratic forms. Amer. J. 51(1929), 51-60.

127. B. Venkov, Über die Arithmetik der Quaternionen. IV. (Russian) Bull. Ac. Sc. Leningrad (7) 2(1929), 535-562.

128. B. Venkov, Über die Arithmetik der Quaternionen. V. (Russian) Bull. Ac. Sc. Leningrad (7) 2(1929), 607-622.

129. T. V. Vepkhvadze, On a formula of Ja. V. Uspenski1. (Russian) Soobshch. Akad. Nauk Gruzin. SSR 46(1967), No. 2, 301-308.

130. T. V. Vepkhvadze, On certain Liouville formulae. (Russian) Soobshch. Akad. Nauk Gruzin. SSR 40(1965), 279-286.

131. T. V. VepkhVadze, The representation of numbers by positive Gaussian binary quadratic forms. (Russian) Soobshch. Akad. Nauk Gruzin. SSR 56(1969), No, 2, 277-280.

132. T. V. VepkhVAdze, The representation of numbers by positive binary quadratic forms with odd discriminant. (Russian) Soobshch. Akad. Nauk Gruzin. SSR 58(1970), No. 1, 29-32.

133. T. V. VePKhVADZE, The representation of numbers by certain binary quadratic forms. (Russian) Trudy Tbiliss. Univ. A 1 (137)(1971), 17-24.

134. T. V. VepkhVADze, representation of numbers by certain quadratic forms with six variables. (Russian) Trudy Tbiliss. Mat. Inst. Razmadze 40(1971), 5-20.

135. T. V. VepkhVAdze, The representation of numbers by positive Gaussian binary quadratic forms. (Russian) Trudy Tbiliss. Mat. Inst. Razmadze 40(1971), 21-58.

136. T. V. VePkHVADZE, The representation of numbers by positive binary quadratic forms of odd discriminant. (Russian) Trudy Tbiliss. Mat. Inst. Razmadze 45(1974), 5-40.

137. T. V. VepkhVADze, The number of representations of numbers by certain quaternary quadratic forms. (Russian) Trudy Tbiliss. Mat. Inst. Razmadze 45(1974), 41-59.

138. T. V. VepkhVadze, The number of representations of numbers by genera of positive binary quadratic forms. (Russian) Collection of articles on number theory, 3. Questions 
on the representation of number by quadratic forms. Trudy Tbiliss. Mat. Inst. Razmadze $\mathbf{5 7}(1977), 29-39$.

139. T. V. Vepkhvadze, On a formula of Siegel. (Russian) Acta Arith. 40(1981/82), No. $2,125-142$.

140. T. V. VepkhVadze, On the analytic theory of quadratic forms. (Russian) Trudy Tbiliss. Mat. Inst. Razmadze 72(1983), 12-31.

141. T. V. VepkhVAdze, Generalized theta-functions with characteristics and the representation of numbers by positive quadratic forms. (Russian) Soobshch. Akad. Nauk Gruzin. SSR 128(1987), No. 3, 465-468 (1988).

142. T. V. VepkhVADZE, Representation of numbers by positive quadratic forms with five variables. (Russian) Trudy Tbiliss. Mat. Inst. Razmadze 84(1987), 21-27.

143. T. V. Vepkhvadze, Generalized theta functions with characteristics, and representation of numbers by quadratic forms. (Russian) Acta Arith. 53(1990), No. 5, 433-451.

144. T. VepkHVAdze, Modular properties of theta-functions and representation of numbers by positive quadratic forms. Georgian Math. J. 4(1997), No. 4, 385-400.

145. T. Vepkhvadze and N. Tsalugelashvili, On the representation of numbers by certain quadratic forms in ten variables. Proceedings of the 2nd international conference in honour of J. Kubilius, Palanga, Lithuania, September 23-27, 1996. New trends in probability and statistics, Vol. 4, 45-55, VSP, Utrecht, 1997.

146. A. Walfisz, Zur additiven Zahlentheorie. V. Trudy Mat. Inst. Razmadze 5(1938), 69-114.

147. A. Walfisz, Zur additiven Zahlentheorie. VI. Trudy Mat. Inst. Razmadze 5(1938), 197-254.

148. A. Walfisz, Zur additiven Zahlentheorie. VIII. Trudy Mat. Inst. Razmadze 8(1940), 69-107.

149. A. Walfisz, Zur additiven Zahlentheorie. IX. Trudy Mat. Inst. Razmadze 9(1941), 75-96.

150. G. L. WATson, Integral quadratic forms. Cambridge Tracts in Mathematics and Mathematical Physics, No. 51. Cambridge University Press, New York, 1960.

(Received 1.11.2006) $)^{1}$

\footnotetext{
${ }^{1}$ This is in fact the English translation of one of the chapters from George Lomadze's booklet "Main results of the research in number theory done in Georgia" published in the Georgian language (Tbilisi University Press, Tbilisi, 2000, 76 pages). A slightly revised Russian version of this survey appeared in the Proceedings of Tbilisi University (Trudy Tbiliss. Univ.), v. 346, 2003, pp. 7-55. Regretfully, the author did not provide the survey with bibliography. The English translation and the list of references have been prepared by N. Kachakhidze in cooperation with a group of other former students of G. Lomadze.
} 